\title{
BIMEASURABLE FUNCTIONS
}

\author{
R. DANIEL MAULDIN ${ }^{1}$
}

\begin{abstract}
Let $X$ and $Y$ be complete, separable metric spaces, $B$ a Borel subset of $X$ and $f$ a Borel measurable map of $B$ into $Y$. The map $f$ is said to be bimeasurable if the image of every Borel subset of $B$ is a Borel subset of $Y$. R. Purves proved that $f$ is bimeasurable if and only if $\left\{y: f^{-1}(y)\right.$ is uncountable $\}$ is countable. The purpose of this note is to demonstrate how Purves' theorem can be obtained directly from some results concerning parametrization of sets which have been developed in the past few years.
\end{abstract}

Let us make the following convention: if $T \subset H \times K$ and $k \in K$, then $T^{k}=\{h$ $\in H:(h, k) \in T\}$. Our first theorem is an easy application of some parametrization results [2], [3].

THEOREM 1. Let $A$ be an analytic subset of $X \times Y$ so that $U=\left\{y: A^{y}\right.$ is uncountable $\}$ is uncountable. Then there is a nonempty compact perfect subset $P$ of $U$ and a Borel isomorphism $g$ of $\{0,1\}^{N} \times P$ onto a subset $R$ of $A$ so that for each $p$ in $P, g \mid\{0,1\}^{N} \times\{p\}$ is a homeomorphism onto $R^{p} \times\{p\}$.

Before proving Theorem 1, let us show how Purves' theorem follows directly from Theorem 1.

Theorem 2 (PURves [4]). Let $B$ be a Borel subset of $X$ and $f$ a Borel measurable map of $B$ into $Y$. In order that $f$ be bimeasurable it is necessary and sufficient that $\left\{y \in Y: f^{-1}(y)\right.$ is uncountable $\}$ is countable.

Proof. Sufficiency is an immediate consequence of a theorem of Lusin [1].

In order to show necessity, suppose that $U=\left\{y: f^{-1}(y)\right.$ is uncountable $\}$ is uncountable. Then $A=\operatorname{Gr}(f)$ is an analytic subset of $X \times Y$ so that $U=\left\{y: A^{y}\right.$ is uncountable $\}$ is uncountable. Let $P, g$ and $R$ be as in Theorem 1. Let $H$ be a Borel subset of $\{0,1\}^{N} \times P$ whose projection, $J$, into $P$ is not a Borel set. Let $L=\pi_{X}(g(H))$. Since $\pi_{X} \mid \operatorname{Gr}(f)$ is one-to-one, $L$ is a Borel subset of $B$. Since $f(L)=J, f$ is not bimeasurable. Q.E.D.

We recall the following notation from [2], [3]. The family of $C$-sets of Selivanovskii, the smallest family of subsets of $X \times Y$ containing the open sets, closed under operation $A$, and closed under complementation is denoted by $S(X \times Y)$. This is a family of universally measurable sets.

Received by the editors May 20, 1980 and, in revised form, December 2, 1980.

1980 Mathematics Subject Classification. Primary 04A15, 54H05, 28A20; Secondary 54C60.

Key words and phrases. Borel isomorphism, bimeasurable function.

${ }^{1}$ Research supported in part by NSF Grant MCS 7804376 and a Faculty Research Grant from North Texas State University. 
Proof of Theorem 1. Since $U$ is an analytic set [1] and $U$ is uncountable there is a homeomorph, $M$, of the Cantor set contained in $U$. Let $E=A \cap(X \times M)$. Then $E$ is an analytic subset of $X \times M$ such that for each $m \in M, E^{m}$ is uncountable. It follows from Theorem 9 of [2] that there is a map $\phi$ of $X \times M$ onto $E$ so that (1) for each $m \in M, \phi(\cdot, m)$ is a one-to-one map of $X \times\{m\}$ onto $E^{m} \times\{m\}$ and (2) both $\phi$ and $\phi^{-1}$ are $S(X \times M)$-measurable.

Let $\mu$ and $\nu$ be atomless probability measures defined on the family of Borel subset of $X$ and $M$, respectively. Let $\mu \times \nu$ be the associated complete probability measure on $X \times M$. Since $\phi$ is $\mu \times \nu$ measurable, there is a compact subset $K$ of $X \times M$ so that $(\mu \times \nu)(K)>1 / 2$ and $\phi \mid K$ is continuous.

Let $T=\left\{m: \mu\left(K^{m}\right)>0\right\}$. Clearly $\nu(T)>0$. Let $P$ be a compact perfect subset of $T$ so that $\nu(P)>0$. Define $\tau$ on $\mathscr{B}(X) \times P$ by $\tau(B, p)=\mu(B)$. Then $\tau$ is a conditional probability distribution on $\mathscr{B}(X) \times P$ such that for each $p$ in $P$, $\tau\left(K^{p}, p\right)>0$. It follows from Theorem 2.4 of [3] that there is a Borel isomorphism $\beta$ of $\{0,1\}^{N} \times P$ onto a subset $G$ of $K$ so that for each $p$ in $P, \beta(\cdot, p)$ is a homeomorphism of $\{0,1\}^{N} \times\{p\}$ onto $G^{p} \times\{p\}$. Thus, the map $g=\phi \circ \beta$ and the subset $R=\phi(G)$ of $A$ have the required properties. Q.E.D.

Let us point out another line of thought concerning bimeasurable functions. It involves more general set theoretic considerations rather than direct descriptive set theoretic considerations. In [5], Darst proved, assuming $\mathrm{CH}$, that $f$ is bimeasurable if and only if $f(M)$ is a universally measurable subset of $Y$ for each universally measurable subset $M$ of $X$. Mauldin [6] observed that the result of Darst held assuming Martin's Axiom. E. Grzegorek and Ryll-Nardzewski [7] completed this line of reasoning by showing that Darst's theorem held in ZFC. Finally, Grzegorek [8] proved in ZFC that $f$ is bimeasurable if and only if $f(N)$ is a universally null subset of $Y$ for each universally null subset $N$ of $X$.

\section{REFERENCES}

1. K. Kuratowski, Topology, Vol. I, Academic Press, New York, 1966.

2. D. Cenzer and R. D. Mauldin, Measurable parametrizations and selections, Trans. Amer. Math. Soc. 245 (1978), 399-408.

3. R. D. Mauldin, Borel parametrizations, Trans. Amer. Math. Soc., 250 (1979), 223-234.

4. R. Purves, Bimeasurable functions, Fund. Math. 58 (1966), 149-157.

5. R. B. Darst, A characterization of bimeasurable functions in terms of universally measurable sets, Proc. Amer. Math. Soc. 27 (1971), 566-571.

6. R. D. Mauldin, Some effects of set-theoretical assumptions in measure theory, Adv. in Math. 27 (1978), 45-62.

7. E. Grzegorek and C. Ryll-Nardzewski, On universal null sets, Proc. Amer. Math. Soc. 81 (1981), 613-617.

8. E. Grzegorek, On some results of Darst and Sierpinski concerning universal null and universally measurable sets (preprint).

Department of Mathematics, North Texas State University, Denton, Texas 76203 